\title{
Performance Evaluation of High Speed Data Networks with Different Transmission Modes
}

\author{
Anu Chaudhary \\ Associate Professor (IT Dept.) \\ Ajay kumar Garg Engineering College \\ Ghaziabad (U.P) India
}

\begin{abstract}
In this paper, a discrete-time single server model is considered in which the server functions can be interrupted and serves on dual mode operating strategy. After a server interruption, the server can either continue the transmission of the message or can completely retransmit the message. These modes are called Continue After Interruption (CAI) mode and Repeat After Interruption (RAI) mode respectively. Two types of traffic are considered (i) multimedia data traffic server operates on Continuous After Interrupt (CAI) mode and (ii) normal data traffic mode server is at Retransmit after Interrupt (RAI) mode. First, we give some general results on a GI-1-1 queue. Next we derive expression for the probability generating function of system contents and cell delays. Some performance measures such as mean delays have been derived in both types of server modes.
\end{abstract}

\section{Keywords}

Discrete-time queueing model, mean packet delays, server interruption, Continue After Interruption (CAI) mode and Repeat After Interruption (RAI) mode.

\section{INTRODUCTION}

In present communication system, digital computer and communication networks including mobile and B-ISDN have advantages of discrete time queueing environment with server interruption, where the time is slotted. Modern communication networks like ATM, operate on the said strategy. Discrete time queueing theory plays an important role to evaluate the performance of such type of communication system. In high speed data networks different transmission and traffic modes needs different quality of service (QoS) standards.

In resent years there has been much interest in high speed data networks and it is a promising technology for transmission of high bandwidth demanding applications. The initial queueing models were based on investigating continuous-time models, but in recent years studies focus on discrete models with server interruptions. Hsu. J [8] has defined a single server system with Bernoulli server interruptions and a poisson arrival processes. Bruneel [9] discussed a multi-server system with i.i.d.(interdependent and identically distributed ) message arrival and server interruption processes. The delay analysis for this system is presented by Laevens and Bruneel [6]. Bruneel [9], Yang and Mark [7] presented a single-server system with i.i.d arrival and correlated on/off server interruption process. In Yang and Mark [7] the on- and off times are assumed to be a series of i.i.d. shifted geometric random variables, whereas in Bruneel [9] the on- and off times are modeled as a series of generally distributed i.i.d. random variables.
The only restriction in the later contribution is that the common probability generating function of the on-times must be rational. All these models have a fixed message length of one packet in common. Queueing model with server vacations [1] occur when several users have to share a common resource, such as polling systems [2], multi-class queueing system [3] etc. or in an environment where the service can be interrupted due to external causes such as machine repair/maintenance models[4] or processor failures[5]. Li-yong et.al proposes a new analysis model of MAC protocol of wireless communication network, which has made possible the polling service capable of differentiating the stations of different priority levels under mixed services policy and has improved the system's flexibility and adaptability via adjustment of the switch-in service in time of load change input [10]. Sikdar[11] considered the performance of the polling based service classes of IEEE 802.16 based broadband wireless access networks and develops queueing models to evaluate their delay distributions and loss rates. Moon et.al considered a multi-server queueing model with a correct analysis of the model [12].

An approach that relates physical system parameters to the queueing performance of wireless links. The potential benefits of multi-rate techniques such as hybrid-ARQ are assessed in the context of delay-sensitive traffic using large deviations. A continuous-time Markov channel model is employed to partition the instantaneous data-rate received at the destination into a finite number of states, each representing a mode of operation of the hybrid-ARQ scheme, the proposed methodology [13] accounts for the correlation of the wireless channel across time, which is computed in terms of level-crossing rates. Rung et.al [14] propose the predictive multicast polling scheme for medium access control in wireless networks we propose to use Markov reward processes to calculate the exact value of the network throughput. Furthermore, they obtained the average system size, the packet blocking probability, and the average packet delay. [15] Considered a multi server queueing model with novel customer (packet) discipline.

In this paper, a discrete time single server model is considered in which server can be interrupted and serves on dual mode operating strategy. In the proposed model, two types of traffics are considered (i) multimedia data traffic and (ii) normal data traffic, where as server interruption is based on the priority discipline. If priority is given to multimedia data traffic then server is interrupted for normal data traffic and interrupted packets will be transmitted with Continuous after Interruption (CAI) operation mode. Similarly if the priority is given to normal data then multimedia data packets will be interrupted and will be retransmitted using Retransmission after Interruption (RAI) operation mode. We have developed GI-1-1 queueing 
model with dual service mode. The expressions for the probability generating function of system contents and packet delays have also been derived.

\section{MODEL DESCRIPTION}

A discrete-time single server queueing system with infinite buffer space is considered, here time is assumed to be slotted, where one slot equals the transmission time of one packet. Packet size is assumed to be invariable. Two types of traffic classes are considered in the system, namely multimedia data class and normal data class. We have denoted the number of arrival of class $j$ during slot $k$ by $a_{j k}(j=1,2)$. Both types of packet arrivals are assumed to be i.i.d. (interdependent and identically distributed) with probability mass function (pmf) $\mathrm{a}(\mathrm{m}, \mathrm{n})$, where

$\mathrm{a}(\mathrm{m}, \mathrm{n})=$ probability $\left[\mathrm{a}_{1, \mathrm{k}}=\mathrm{m}, \mathrm{a}_{2, \mathrm{k}}=\mathrm{n},\right]$

and joint probability generating function (pgf)

$\mathrm{A}\left(\mathrm{z}_{1}, \mathrm{z}_{2}\right)$,

$$
\mathrm{A}\left(\mathrm{z}_{1}, \mathrm{z}_{2}\right)=\mathrm{E}\left[\mathrm{z}_{1}^{\mathrm{a}_{1}, k} \mathrm{z}_{2}^{\mathrm{a}_{2}, k}\right]
$$

Further, the total number of arriving packets during slot-k is denoted by:

$a_{T, k} \cong a_{1, k}+a_{2, k}$ and its pgf is defined

as $A_{T}(z) \cong E\left[z^{a_{T, k}}\right]=A(z, z)$. The pgf's of the number of arrivals from multimedia data class and normal data class during a slot can be defined as:

$A_{1}(z)=E\left[z^{a_{1, k}}\right]=A(1, z)$

and $A_{2}(z)=E\left[z^{a_{2}, k}\right]=A(z, 1)$, respectively.

From these pgf's the arrival rate of class j i.e. $\lambda_{j}$

can be defined as :

$\lambda_{j}=E\left[a_{j, k}\right]=A_{j}^{\prime}(1)$.

and, the total arrival rate by $\lambda_{T}$ is defined as:

$\lambda_{T}=A_{T}^{\prime}=A_{1}^{\prime}(1)+A_{2}^{\prime}(1)$.

The system has one server that provides the transmission of cells, at a rate of 1 cell per slot. The system is assumed to be stable i.e. $\lambda_{T}<1$.

There are two types of traffic classes with priority in the system which is served by dual mode server. The model has been classified in two cases: In the first case the multimedia data is heavy in comparison to normal data, and it has priority over normal data. In this case, the normal data packets will be interrupted and will be transmitted using CAI operation mode. Since the normal data can be stored for longer period of time as compared to multimedia data, therefore the operation mode is as CAI mode [Fig 1].

In the second case, the normal data is heavy as compared to multimedia data and it has priority over multimedia data. Multimedia data packets will be interrupted and service mode will be RAI mode and packets will be entirely retransmitted [Fig 2].

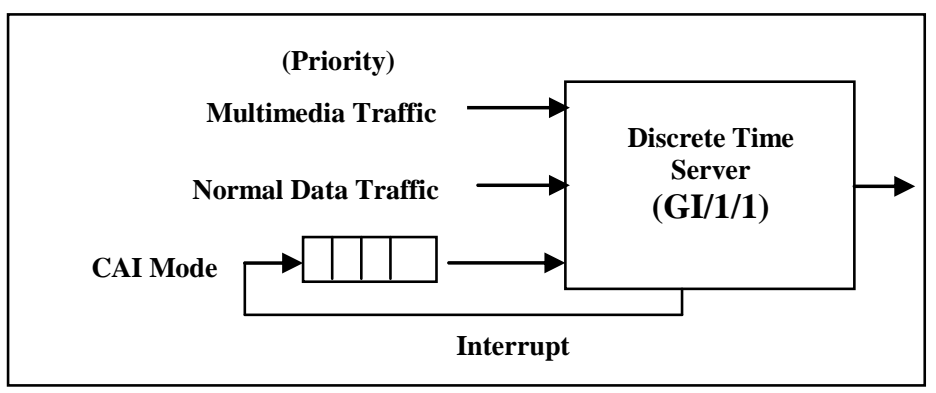

Fig 1: Case-I (Server at CAI Mode)

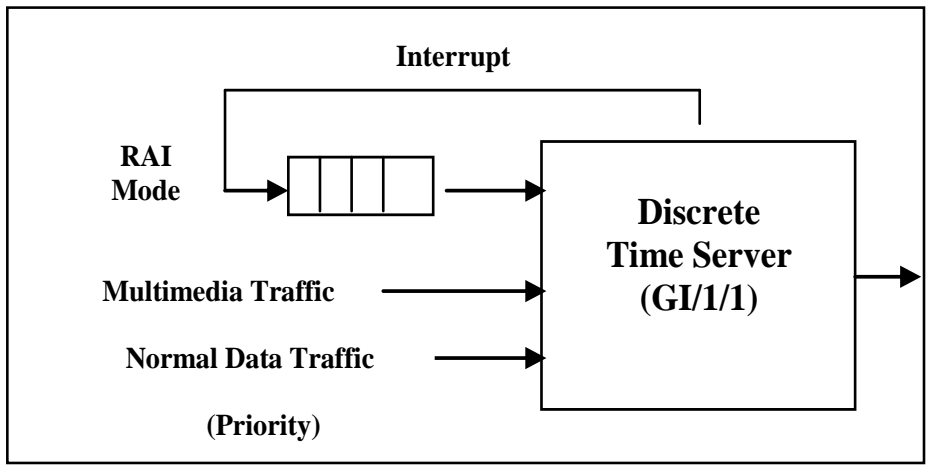

Fig 2: Case-II (Server at RAI Mode)

\section{SYSTEM CONTENTS}

In this section, the probability generating function of the steadystate system contents of both data classes have been obtained, which represent the number of packets in the system. The system contents of class- $\mathrm{j}$ at the beginning of slot $\mathrm{k}$ is defined by $u_{j, k}(j=1,2)$. The pgf of both the data classes are defined by $\mathrm{U}_{k}\left(z_{1}, z_{2}\right)=E\left[z_{1}^{u_{1, k}} z_{2}^{u_{2, k}}\right]$.

\subsection{Case-I: Steady-state System Contents for Heavy Multimedia Data and Light Normal} Data.

In this case priority is given to the heavy data (Multimedia data) and the mode of the operation of the server is CAI. The equations of system contents are as given below:

$$
\begin{aligned}
& \text { If, } U_{1, k}>0: \\
& U_{1, k+1}=\left[u_{1, k}-1\right]^{+}+a_{1, k} \\
& U_{2, k+1}=\left[P_{C A I}-1\right]+u_{2, k}+a_{1, k}+a_{2, k}
\end{aligned}
$$

where, $[\ldots]^{+}$denotes maximum argument and zero, $\mathrm{P}_{\mathrm{CAI}}$ denotes the number of packets to be transmitted using CAI operation mode. 


\subsection{Case-II : Steady- state System} Contents for Heavy Normal Data and Light Multimedia Data.

In this case, priority is given to normal data and the mode of the operation of the server is RAI. The equations of system contents are as given below:

$$
\begin{gathered}
\text { If, } \quad U_{2, k}>0: \\
U_{1, k+1}=\left[P_{R A I}-1\right]+u_{1, k}+a_{1, k}+a_{2, k} \\
U_{2, k+1}=\left[u_{2, k}-1\right]^{+}+a_{2, k}
\end{gathered}
$$

where, $P_{R A I}$ denotes the number of packets to be retransmitted using RAI operation mode.

Calculation of the Probability Generating Function at the beginning of slot $\mathrm{k}+1$,

For Case-I: $\quad U_{k+1}\left(z_{1}, z_{2}\right)=E\left[z_{1}^{u_{1, k+1}} z_{2}^{u_{2, k+1}}\right]$

$$
=E\left[z_{1}^{u_{1, k}-1+a_{1, k}} z_{2}^{P_{C A I}-1+u_{2, k}+a_{1, k}+a_{2, k}}\right]
$$

Using the system equations, the following relation between $U_{k+1}$ and $U_{k}$ is defined as below:

$U_{k+1}\left(z_{1}, z_{2}\right)=U_{k}(0,0)+$

$z_{1}^{-1}\left[U_{k}\left(z_{1}, z_{2}\right)-U_{k}\left(0, z_{2}\right)\right] A\left(z_{1}, z_{2}\right) N_{C A I} A_{T}(1)$

$z_{1} U_{k+1}\left(z_{1}, z_{2}\right)=z_{1} U_{k}(0,0)+$

$\left[U_{k}\left(z_{1}, z_{2}\right)-U_{k}\left(0, z_{2}\right)\right] A\left(z_{1}, z_{2}\right) N_{C A I} A_{T}(1)$

The steady-state distribution of the system contents, can be defined as $U\left(z_{1}, z_{2}\right)$ as:

$$
\begin{aligned}
& U\left(z_{1}, z_{2}\right)=\lim _{k \rightarrow \alpha} U_{k}\left(z_{1}, z_{2}\right) \\
& z_{1} U\left(z_{1}, z_{2}\right)=z_{1} U(0,0)+ \\
& {\left[U\left(z_{1}, z_{2}\right)-U\left(0, z_{2}\right)\right] A\left(z_{1}, z_{2}\right) N_{C A I} A_{T}(1)} \\
& U\left(z_{1}, z_{2}\right)=\frac{z_{1} U(0,0)-U\left(0, z_{2}\right) A\left(z_{1}, z_{2}\right) N_{C A I} A_{T}(1)}{z_{1}-A\left(z_{1}, z_{2}\right) N_{C A I} A_{T}(1)}
\end{aligned}
$$

Similarly, the system contents for case-II can be expressed as

$$
U\left(z_{1}, z_{2}\right)=\frac{z_{2} U(0,0)-U\left(0, z_{1}\right) A\left(z_{1}, z_{2}\right) N_{R A I} A_{T}(2)}{Z_{2}-A\left(z_{1}, z_{2}\right) N_{R A I} A_{T}(2)}
$$

In equation (5) there are two quantities to be determined namely constant $\mathrm{U}(0,0)$ and function $\mathrm{U}\left(0, \mathrm{z}_{2}\right)$. For obtaining the value of $\mathrm{U}\left(0, \mathrm{z}_{2}\right)$, applying Rouche's theorem, It can be shown that for a given value of $z_{2}$, the equation $z_{1}=A\left(z_{1}, z_{2}\right) N_{C A I} A_{T}(1)$ has one solution in the unit circle for $z_{1}$ which will be denoted by $\gamma\left(z_{2}\right)$ i.e. $z_{1}=\gamma\left(z_{2}\right)$. Therefore the equation (5) can be expressed as : $U\left(0, z_{2}\right)=\frac{\gamma\left(z_{2}\right) U(0,0)}{A\left(\gamma\left(z_{2}\right) z_{2}\right) N_{C A I} A_{T}(1)}$

where $U(0,0)$ can be found by applying the normalization condition $\mathrm{U}(1,1)=1$. The de l'hopitals rule gives the expected result for the probability of having an empty system as below:

$U(0,0)=1-\lambda_{T}$

Now putting the values of $U\left(0, z_{2}\right)$ in equation (5).

$$
\begin{aligned}
& U\left(z_{1}, z_{2}\right) \\
& \quad=\frac{z_{1} U(0,0)}{z_{1}-A\left(z_{1}, z_{2}\right) N_{C A I} A_{T}(1)\left[z_{1}-A\left(z_{1}, z_{2}\right) N_{C A I} A_{T}(1)\right] A\left(\gamma\left(z_{2}\right) z_{2}\right)}
\end{aligned}
$$

Similarly, for Case-II

$U\left(z_{1}, z_{2}\right)=\frac{z_{2} U(0,0)}{z_{2}-A\left(z_{1}, z_{2}\right) N_{R A I} A_{T}(2)}-\frac{\gamma\left(z_{1}\right) U(0,0) A\left(z_{1}, z_{2}\right)}{\left[z_{2}-A\left(z_{1}, z_{2}\right) N_{R A I} A_{T}(2)\right] A\left(\gamma\left(z_{1}\right) z_{1}\right)}$

Now calculated pgf of the system contents of $U_{1}(z)$ and $U_{2}(z)$ for Case-I, from equation (8) as given below:

$$
\begin{aligned}
& U_{1}(z)=\lim _{k \rightarrow \infty} E\left[Z^{u_{1, k}}\right]=U(z, 1) \\
& U_{1}(z)=\frac{z U(0,0)}{z-A(z, 1) N_{C A I} A_{T}(1)}-\frac{\gamma(1) U(0,0) A(z, 1)}{z-A(z, 1) N_{C A I} A_{T}(1) A(\gamma(1), 1)}(10) \\
& U_{2}(z)=\lim _{k \rightarrow \infty} E\left[Z^{u_{2, k}}\right]=U(1, z) \\
& U_{2}(z)=\frac{U(0,0)}{1-A(1, z) N_{C A I} A_{T}(1)}-\frac{\gamma(z) U(0,0) A(1, z)}{1-A(1, z) N_{C A I} A_{T}(1) A(\gamma(z), z)}(11)
\end{aligned}
$$

Now, calculating the pgf of the system contents $\mathrm{U}_{1}(\mathrm{z})$ and $\mathrm{U}_{2}(\mathrm{z})$ for Case-II from equation (9):

$$
\begin{aligned}
& U_{1}(z)=\lim _{k \rightarrow \infty} E\left[Z^{u_{1, k}}\right]=U(z, 1) \\
& U_{1}(z)=\frac{U(0,0)}{1-A(z, 1) N_{R A I} A_{T}(2)}-\frac{\gamma(z) U(0,0) A(z, 1)}{1-A(z, 1) N_{R A I} A_{T}(2) A(\gamma(z), z)}(12
\end{aligned}
$$




$$
\begin{aligned}
& U_{2}(z)=\lim _{k \rightarrow \infty} E\left[Z^{u_{2, k}}\right]=U(1, z) \\
& U_{2}(z)=\frac{z U(0,0)}{z-A(1, z) N_{R A I} A_{T}(2)}-\frac{\gamma(1) U(0,0) A(1, z)}{z-A(1, z) N_{R A I} A_{T}(2) A(\gamma(1), 1)}(13)
\end{aligned}
$$

\section{PACKET DELAY}

The packet delay is defined as the total amount of time that a packet spends in the system, i.e., the number of slots between the end of the packets arrival slot and the end of its departure slot. In this section, we derive expressions for the pgf values of the packet's delay of both data traffic classes. The amount of time a tagged class 1 packet spends in the system i.e. Packet delay for multimedia data traffic class is given by: $d_{1}=\left[u_{1, k}-1\right]^{+}+f_{1, k}+1$

where, slot $\mathrm{k}$ is assumed to be the arrival slot of the tagged packet, $u_{1, k}$ is the system contents of multimedia data traffic class at the beginning of slot $\mathrm{k}$ and $f_{1, k}$ is defined as the total number of multimedia class packets that arrive during slot $\mathrm{k}$, and which are to be served before the tagged packet. The tagged class1 packet has to wait (in queue 1) until all the packets that arrived prior to it in this queue are served. The number of such packets is determined by all packets present in queue 1 at the beginning of its arrival slots (which also include class 2 packets which eventually jumped to queue 1 before the arrival of tagged class 1 packet). The delay thus represents the waiting time coupled with the service time of a packet which equals 1 .

Similarly, packet delay for normal data traffic class is given by:

$d_{2}=N_{C A I}+u_{2, k}+f_{1, k}+f_{2, k}$

and $u_{2, k}$ is the system contents of normal data traffic class at the beginning of slot $\mathrm{k}$ and $f_{2, k}$ is defined as the total number of normal class packets that arrive during slot $\mathrm{k}$.

(Similarly for Case-II)

Packet delays for multimedia data traffic class

$d_{1}=N_{R A I}+u_{1, k}+f_{1, k}+f_{2, k}$

Packet delays for normal data traffic class

$d_{2}=\left[u_{2, k}-1\right]^{+}+f_{2, k}+1$

In this section the packet delay of multimedia data traffic class packets is being analyzed as if they are the only type of packets in the system. The pgf of the packet delay of multimedia data traffic class (delay after interruption) is given by:
For Case-I,

$$
\begin{aligned}
& D_{1}(z)=E\left[z^{d_{1}}\right] \\
& \mathrm{D}_{1}(\mathrm{z})=\mathrm{F}_{1}(z)\left[U_{1}(z)-(z-1) U_{1}(0)\right]
\end{aligned}
$$

The pgf $F_{1}(z)=E\left[z^{f_{1}, k}\right]$ can be calculated by taking into account that an arbitrary packet is arriving in bulk [Bruneel 93]:

$$
\text { where, } \quad F_{1}(z)=\frac{A_{1}(z)-1}{\lambda_{1}(z-1)}
$$

Using expressions (10) and (19) in (18)

$\mathrm{D}_{1}(\mathrm{z})=\frac{A_{1}(z)-1}{\lambda_{1}(z-1)}\left[\frac{z U(0,0)}{z-A(z, 1) N_{C A I} A_{T}(1)}-\frac{\gamma(1) U(0,0) A(z, 1)}{z-A(z, 1) N_{C A I} A_{T}(1) A(\gamma(1), 1)}\right](20)$ $-\frac{\left(A_{1}(z)-1\right)(z-1) \gamma(1) U(0,0) A(0,1)}{\lambda_{1}(z-1) A(0,1) N_{C A l} A_{T}(1) A(\gamma(1), 1)}$

The pgf of the packet delay of normal data traffic class (delay after interruption) is given by:

$$
\begin{aligned}
& \begin{array}{l}
D_{2}(z)=E\left[z^{d_{2}}\right] \\
D_{2}(z)=N_{C A I} \times F_{1}(z) \times F_{2}(z) \times U_{2}(z)
\end{array} \\
& \text { where, } F_{2}(z)=\frac{A_{2}(z)-1}{\lambda_{2}(z-1)}
\end{aligned}
$$

Using expressions (11) and (22) in (21)

$$
\begin{aligned}
& D_{2}(z)=N_{C A I} \times \frac{\left(A_{1}(z)-1\right)}{\lambda_{1}(z-1)} \times \frac{\left(A_{2}(z)-1\right)}{\lambda_{2}(z-1)} \\
& {\left[\frac{U(0,0)}{1-A(1, z) N_{C A I} A_{T}(1)}-\frac{\gamma(z) U(0,0) A(1, z)}{1-A(1, z) N_{C A I} A_{T}(1) A(\gamma(z), z)}\right]}
\end{aligned}
$$

Similarly, for Case-II

$$
\begin{aligned}
& D_{1}(z)=N_{R A I} \times F_{1}(z) \times F_{2}(z) \times U_{1}(z) \\
& D_{1}(z)=N_{R A I} \times \frac{\left(A_{1}(z)-1\right)}{\lambda_{1}(z-1)} \times \frac{\left(A_{2}(z)-1\right)}{\lambda_{2}(z-1)} \\
& {\left[\frac{U(0,0)}{1-A(z, 1) N_{R A I} A_{T}(2)}-\frac{\gamma(z) U(0,0) A(z, 1)}{1-A(z, 1) N_{R A I} A_{T}(2) A(\gamma(z), z)}\right]} \\
& \mathrm{D}_{2}(\mathrm{z})=\mathrm{F}_{2}(z)\left[U_{2}(z)-(z-1) U_{2}(0)\right]
\end{aligned}
$$

$$
\begin{aligned}
& D_{2}(z)=\frac{A_{2}(z)-1}{\lambda_{2}(z-1)}\left[\frac{z U(0,0)}{z-A(1, z) N_{R A I} A_{T}(2)}-\frac{\gamma(1) U(0,0) A(1, z)}{z-A(1, z) N_{R A I} A_{T}(2) A(\gamma(1), 1)}\right] \\
& -\frac{\left(A_{2}(z)-1\right)(z-1) \gamma(1) U(0,0) A(1,0)}{\lambda_{2}(z-1) A(1,0) N_{R A I} A_{T}(2) A(\gamma(1), 1)}
\end{aligned}
$$




\section{CALCULATION OF MEAN OF PACKET DELAY}

In this section the expression for mean of packet delay for both types of traffic classes are derived. This can be achieved by taking their derivatives of respective pgf for $\mathrm{z}=1$.

The mean packet delays for case-I is calculated below:

The pgf of the packet delay of multimedia data is given by

$\mathrm{D}_{1}(\mathrm{z})=\frac{A_{1}(z)-1}{\lambda_{1}(z-1)}\left[\frac{z U(0,0)}{z-A(z, 1) N_{C A I} A_{T}(1)}-\frac{\gamma(1) U(0,0) A(z, 1)}{z-A(z, 1) N_{C A I} A_{T}(1) A(\gamma(1), 1)}\right]$ $-\frac{\left(A_{1}(z)-1\right)(z-1) \gamma(1) U(0,0) A(0,1)}{\lambda_{1}(z-1) A(0,1) N_{C A I} A_{T}(1) A(\gamma(1), 1)}$

$\mathrm{D}_{1}(\mathrm{z})=\frac{\mathrm{z}\left(\mathrm{A}_{1}(\mathrm{z})-1\right) \mathrm{U}(0,0) \mathrm{A}(\gamma(1), 1)-\gamma(1) \mathrm{U}(0,0) \mathrm{A}(\mathrm{z}, 1)\left(\mathrm{A}_{1}(\mathrm{z})-1\right)}{\lambda_{1}(z-1) A(\gamma(1), 1)\left[z-A(z, 1) N_{C A I} A_{T}(1)\right]}$ $-\frac{\left(A_{1}(z)-1\right)(z-1) \gamma(1) U(0,0) A(0,1)}{\lambda_{1}(z-1) A(0,1) N_{C A} A_{T}(1) A(\gamma(1), 1)}$

Putting $\quad A_{T}(1)=1, A(\gamma(1), 1)=1, \gamma(1)=1$ in the above equation:

$D_{1}(\mathrm{z})=\frac{\mathrm{z}\left(\mathrm{A}_{1}(\mathrm{z})-1\right) \mathrm{U}(0,0)-\mathrm{U}(0,0) \mathrm{A}(\mathrm{z}, 1)\left(\mathrm{A}_{1}(\mathrm{z})-1\right)}{\lambda_{1}(z-1)\left(z-A(z, 1) N_{C A I}\right)}-\frac{\left(A_{1}(z)-1\right)(z-1) U(0,0)}{\lambda_{1}(z-1) N_{C A I}}$

$\lambda_{1}(z-1) D_{1}(\mathrm{z})$

$=\frac{\mathrm{z}\left(\mathrm{A}_{1}(\mathrm{z})-1\right) \mathrm{U}(0,0) \mathrm{N}_{\mathrm{CAI}}-U(0,0) A(z, 1)\left(A_{1}(z)-1\right) N_{C A I}-\left(A_{1}(z)-1\right)(z-1) U(0,0)[z-A(z, 1)] N_{C A I}}{\left(z-A(z, 1) N_{C I}\right) N_{C H}}$ $\left(z-A(z, 1) N_{C A I}\right) N_{C A I}$

$\lambda_{1}(z-1)\left(z-A(z, 1) N_{C A I}\right) N_{C A I} D_{1}(z)=\mathrm{z}\left(\mathrm{A}_{1}(\mathrm{z})-1\right) \mathrm{U}(0,0) \mathrm{N}_{\mathrm{CAI}}-U(0,0) A(z, 1)\left(A_{1}(z)-1\right) N_{C A I}$ - $\left(A_{1}(z)-1\right)(z-1) U(0,0)[z-A(z, 1)] N_{C A I}$

Differentiating w.r.t. $\mathrm{z}$ as given and putting, $\mathrm{z}=1$ and $A_{1}^{\prime}(1)=\lambda_{1}$, we get:

$D_{1}^{\prime}(1)=\frac{\left(1-\lambda_{T}\right)}{2\left(1-N_{C A I}\right)}-\frac{\left(1-\lambda_{T}\right) A^{\prime}(1,1)}{2\left(1-N_{C A I}\right)}-\frac{\left(1-\lambda_{T}\right)}{N_{C A I}}-\frac{\left[1-A^{\prime}(1,1) N_{C A I}-N_{C A I}^{\prime}\right]}{\left(1-N_{C A I}\right)}-\frac{N_{C A I}^{\prime}}{N_{C A I}}$ Putting $U(0,0)=\left(1-\lambda_{T}\right)$, The expression for mean delay for class 1 i.e. $D_{1}^{\prime}(1)$ is defined as:

$D_{1}^{\prime}(1)=\frac{\left(1-\lambda_{T}\right)}{2\left(1-N_{C A I}\right)}-\frac{\left(1-\lambda_{T}\right) A^{\prime}(1,1)}{2\left(1-N_{C A I}\right)}-\frac{\left(1-\lambda_{T}\right)}{N_{C A I}}-\frac{\left[1-A^{\prime}(1,1) N_{C A I}-N_{C A I}^{\prime}\right]}{\left(1-N_{C A I}\right)}-\frac{N_{C A I}^{\prime}}{N_{C A I}}$

The pgf of the packet delay of normal data can be calculated by differentiating w.r.t $\mathrm{z}$ and putting $\mathrm{z}=1$, as given below:

$$
\begin{aligned}
D_{2}^{\prime}(1)= & \frac{2 N_{C A I} \lambda_{22} U(0,0)}{3 \lambda_{2}\left[1-N_{C A I}\right]}-\frac{N_{C A I}^{\prime} U(0,0)}{3\left[1-N_{C A I}\right]}+\frac{N_{C A I} U(0,0) A^{\prime}(1)}{\left[1-N_{C A I}\right]}+\frac{N_{C A I} \lambda_{11} U(0,0)}{6 \lambda_{1}\left[1-N_{C A I}\right]} \\
& -\frac{5 N_{C A I} \gamma^{\prime}(1) U(0,0)}{6\left[1-N_{C A I}\right]}-\frac{N_{C A I} U(0,0) A^{\prime}(1,1)}{\left[1-N_{C A I}\right]}-\frac{N_{C A I} U(0,0)}{6\left[1-N_{C A I}\right]} \\
& +\frac{\left[A^{\prime}(1,1) N_{C A I}+N_{C A I}^{\prime}\right]}{\left[1-N_{C A I}\right]}-A^{\prime}(1)
\end{aligned}
$$

mean delay for class2 i.e. $D_{2}^{\prime}(1)$ is as given, by putting $U(0,0)=1-\lambda_{T}$, as follows:

$$
\begin{aligned}
D_{2}^{\prime}(1)= & \frac{2 N_{C A I} \lambda_{22}\left(1-\lambda_{T}\right)}{3 \lambda_{2}\left[1-N_{C A I}\right]}-\frac{N_{C A I}^{\prime}\left(1-\lambda_{T}\right)}{3\left[1-N_{C A I}\right]}+\frac{N_{C A I}\left(1-\lambda_{T}\right) A^{\prime}(1)}{\left[1-N_{C A I}\right]}+\frac{N_{C A I} \lambda_{11}\left(1-\lambda_{T}\right)}{6 \lambda_{1}\left[1-N_{C A I}\right]} \\
& -\frac{5 N_{C A I} \gamma^{\prime}(1)\left(1-\lambda_{T}\right)}{6\left[1-N_{C A I}\right]}-\frac{N_{C A I}\left(1-\lambda_{T}\right) A^{\prime}(1,1)}{\left[1-N_{C A I}\right]}-\frac{N_{C A I}\left(1-\lambda_{T}\right)}{6\left[1-N_{C A I}\right]} \\
& +\frac{\left[A^{\prime}(1,1) N_{C A I}+N_{C A I}^{\prime}\right]}{\left[1-N_{C A I}\right]}-A^{\prime}(1)
\end{aligned}
$$

Similarly, for Case II the mean delays $D_{1}^{\prime}(1)$ and $D_{2}^{\prime}(1)$ are given by:

$$
\begin{aligned}
D_{1}^{\prime}(1)= & \frac{\left(1-\lambda_{T}\right)}{2\left(1-N_{R A I}\right)}-\frac{\left(1-\lambda_{T}\right) A^{\prime}(1,1)}{2\left(1-N_{R A I}\right)}-\frac{\left(1-\lambda_{T}\right)}{N_{R A I}}-\frac{\left[1-A^{\prime}(1,1) N_{R A I}-N_{R A I}^{\prime}\right]}{\left(1-N_{R A I}\right)}-\frac{N_{R A I}^{\prime}}{N_{R A I}} \\
D_{2}^{\prime}(1)= & \frac{2 N_{R A I} \lambda_{22}\left(1-\lambda_{T}\right)}{3 \lambda_{2}\left[1-N_{R A I}\right]}-\frac{N_{R A I}^{\prime}\left(1-\lambda_{T}\right)}{3\left[1-N_{R A I}\right]}+\frac{N_{R A I}\left(1-\lambda_{T}\right) A^{\prime}(1)}{\left[1-N_{R A I}\right]}+\frac{N_{R A I} \lambda_{11}\left(1-\lambda_{T}\right)}{6 \lambda_{1}\left[1-N_{R A I}\right]} \\
& -\frac{5 N_{R A I} \gamma^{\prime}(1)\left(1-\lambda_{T}\right)}{6\left[1-N_{R A I}\right]}-\frac{N_{R A I}\left(1-\lambda_{T}\right) A^{\prime}(1,1)}{\left[1-N_{R A I}\right]}-\frac{N_{R A I}\left(1-\lambda_{T}\right)}{6\left[1-N_{R A I}\right]} \\
& +\frac{\left[A^{\prime}(1,1) N_{R A I}+N_{R A I}^{\prime}\right]}{\left[1-N_{R A I}\right]}-A^{\prime}(1)
\end{aligned}
$$

\section{NUMERICAL EXAMPLE}

In this section, the results obtained in the previous sections are applied. Two types of traffic are assumed with priority and dual server operation mode. Traffic of class-1 is delay sensitive (for video) and in this order traffic of class- 2 is assumed to be delay insensitive (for instance data). The packet arrivals on each epoch are assumed to be i.i.d. with total arrival rate $\lambda_{T}$. Interrupted packets in Case-I are assumed to be arriving at slot $\mathrm{k}$ by Bernoulli process hence their pgf will be defined by:

$N_{C A I}=\left[1-\frac{\lambda_{2}}{2}\left(1-z_{2}\right)\right]^{2}, N_{C A I}^{\prime}=\lambda_{2}\left[1-\frac{\lambda_{2}}{2}\left(1-z_{2}\right)\right]$

Similarly for case -II,

$$
N_{R A I}=\left[1-\frac{\lambda_{1}}{2}\left(1-z_{1}\right)\right]^{2}, \quad N_{R A I}^{\prime}=\lambda_{1}\left[1-\frac{\lambda_{1}}{2}\left(1-z_{1}\right)\right]
$$

Where $\alpha$ is defined as the fraction of class- 1 arrivals in overall traffic mix (i.e. $\alpha=\frac{\lambda_{1}}{\lambda_{T}}$ ). The mean packet delays for case-I and case-II, when $\alpha=0.10,0.50$ and 0.75 are shown in Fig 3 and Fig 4, respectively. 


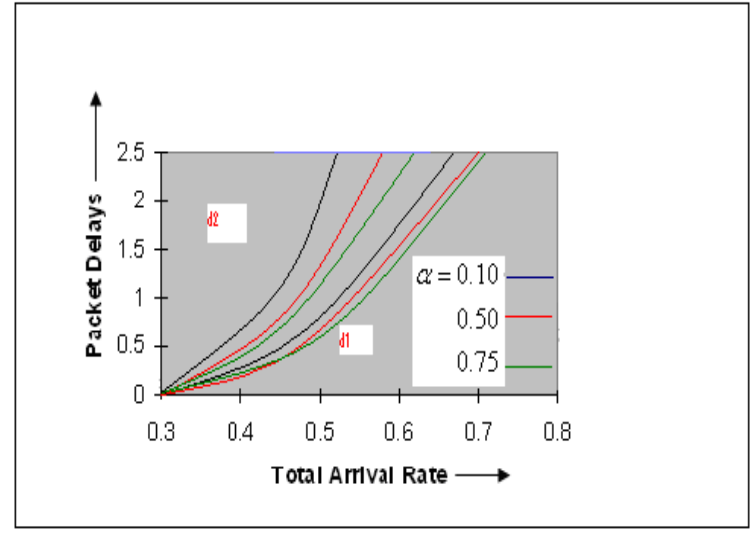

Fig 3: Packet Delays Vs Total Arrival Rates (Case-I)

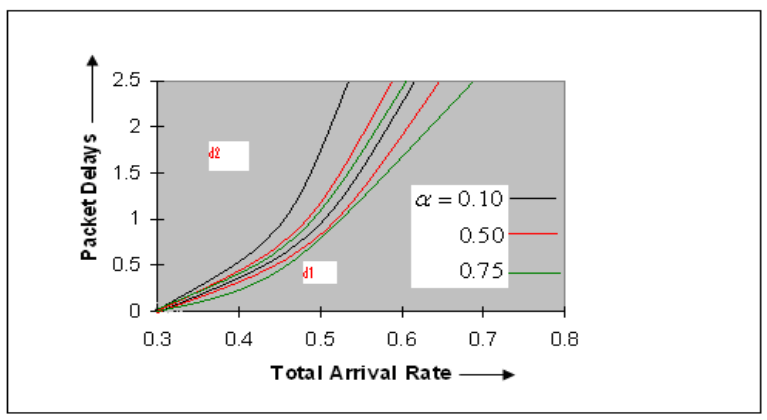

Fig 4: Packet Delays Vs Total Arrival Rates (Case-II)

\section{CONCLUSION}

In this paper a discrete time single server model with server interruption and dual operation mode is discussed, where two types of data traffic with priority discipline are considered. When multimedia data traffic is heavy then it has priority over normal data traffic class and in this case service of normal data traffic class will be interrupted and interrupted and packets will be retransmitted using CAI mode. Here the operation mode is CAI because normal data packets can be restored for a longer period. In Case-II when normal data traffic is heavy then it has priority over multimedia data traffic class and in this case service will be interrupted for this class and interrupted packets will be entirely retransmitted using RAI mode. Mean packet delay for both operation modes are evaluated. Graphs (Fig 3 and Fig 4) indicates that packet delay using CAI mode is less than RAI mode. Therefore results show that this model can be helpful where server is interrupted (like mobile and wireless networks) and operation mode is dual.

\section{REFERENCES}

[1]. Doshi B.T.,"Queueing system with vacations-a survey", Queueing System(1) pp 29-66,1986.

[2]. Takagi H.A.,"A survey of queueing analysis of polling models", In Proceedings of the Third IFIP International
Conference on Data Communication systems and their Performance, Rio de Janeiro, Brazil pp 22-25, June 1987.

[3]. Coury S, Harrison PG, "Waiting time distribution in a class of discrete-time cyclic service multi-queue systems", Performance Evaluation (29) pp 223-43, 1997.

[4]. Van der Duyn Schouten FA, Vanneste SG, "Maintenance optimization of a production system with buffer capacity", European Journal of Operation Research pp 232-338, 1995.

[5]. Altiok T. "Queueing modeling of a single processor with failures", Performance Evaluation pp 93-102, 1988/89.

[6]. Laevens K, Bruneel H, "Delay analyses for discrete-time queueing systems with multiple randomly interrupted servers", European Journal of Operational Research (85) pp 161-77, 1995.

[7]. Yang OWW, Mark JW, "Performance analysis of integrated services on a single server system", Performance Evaluation (11) pp 79-92, 1990.

[8]. Hsu J., "Buffer behavior in computer communication system", IEEE transactions on Communications (22) pp 1940-41, 1974.

[9]. Bruneel H.A, "A general model for the behavior of infinite buffers with periodic service opportunities", European Journal of Operation Research (16), pp 98-106, 1984.

[10]. Li-Yong, Dong-Feng, Hong-Wei, "The Analysis of the MAC Protocol of Two-Class Priority Station Polling System under Mixed Services Policy of Exhaustive and Two-Times Gated Services", IEEE Computer Society, Second International conference on Networks Security, Wireless Communications and Trusted Computing, pp 344347,2010

[11]. Sikdar, B., "Queueing analysis of polled service classes in the IEEE 802.16 MAC protocol", IEEE Transactions on Wireless Communications (8), pp 57-67, 2009.

[12]. Moon Ho Lee, Alexander Dudin, Valentina Klimenok, "Mathematical analysis of the multi-server queueing model for dynamic channel reservation in wireless networks", IEEE Communications Letters (10), pp 855-857, 2006.

[13].Gunaseelan, Nirmal, Lingjia Liu Chamberland, Gregory H. Huff "Performance analysis of wireless hybrid-ARQ systems with delay-sensitive Traffic", IEEE Transactions on Communications (4), pp 1262-1272,2010.

[14].Rung-HungGau,Kuan-MeiChen, "Predictive multicast polling for wireles networks with multipacket reception and queuing", IEEE Transactions on Mobile Computing (5), $\quad$ pp 725-737,2006.

[15]. Moon Ho Lee, Alexander Dudin, Valentina Klimenok, Chang Joa Kim, "Multi-Server Queueing Model with Broadcasting Service, IEEE Communications Letters (11), pp 546-548, 2007. 